\title{
Isolation and Host Range of Lytic Staphylophages on Clinical Isolates of Methicillin-Resistant Staphylococcus aureus
}

\author{
Mahtab Sadat Madani Boroujeni ${ }^{1}$, Mohammadreza Mahzounieh (iD ${ }^{1,2,}{ }^{*}$, Azizollah Ebrahimi \\ Kahrizsangi ${ }^{1}$, Soudabeh Rostami ${ }^{2}$, Azam Mokhtari ${ }^{1}$, Somaye Gheisarbeigi ${ }^{1}$ and Saeid \\ Amirizadehfard ${ }^{1,3}$ \\ ${ }^{1}$ Department of Pathobiology, Shahrekord University, Shahrekord, Iran \\ ${ }^{2}$ Research Center for Infectious Diseases and Tropical Medicine, Isfahan University of Medical Sciences, Isfahan, Iran \\ ${ }^{3}$ Department of Immunology, Shiraz University of Medical Sciences, Shiraz, Iran \\ "Corresponding author: Department of Pathobiology, Shahrekord University, Shahrekord, Iran. Email: mahzounieh@sku.ac.ir
}

Received 2020 August 24; Revised 2020 October 04; Accepted 2020 October 06.

\begin{abstract}
Background: Staphylococcus aureus (S. aureus) is a major cause of nosocomial infections in humans and animals. Because of the widespread resistance to antibiotics, microbiologists are trying to find other therapeutic interventions such as phage therapy for bacterial infections.

Objectives: The present study aimed to isolate staphylophages with lytic effects on methicillin-resistant S. aureus (MRSA) clinical isolates as a potential alternative agent to antibiotic therapy.

Methods: This experimental, descriptive study is performed in the Microbiology Laboratory of Shahrekord University (Iran) from September 2018 to March 2019. Two cocktails of staphylophages were isolated from Isfahan (Iran) urban sewage samples. The doublelayer agar method was used to detect lytic phages. Morphology characteristic by transmission electron microscopy (TEM) images was used to identify staphylophages. One hundred and thirty three $S$. aureus were isolated from clinical samples of two teaching hospitals in Isfahan and Shiraz, Iran. Methicillin resistance and the presence of the mecA gene were determined by the disk diffusion method and polymerase chain reaction (PCR) assay, respectively. The phage susceptibility of mecA positive isolates was determined by plaque assay.

Results: Two staphylophage cocktails were prepared, which had lytic effects on forty-four MRSA isolates. Cocktails 1 and 2 lysed 19 $(14.2 \%)$ and 25 (18.7\%) isolates, respectively. Of 133 S. aureus isolates, $88.7 \%$ carried the mecA gene.

Conclusions: Different bacteriophages in two phage cocktails had relatively good lytic effects on S. aureus clinical isolates. Therefore, phage cocktails may be an appropriate alternative to antibiotics against S. aureus.
\end{abstract}

Keywords: Bacteriophages, Methicillin-Resistant, Staphylococcus aureus, mecA Gene

\section{Background}

Staphylococcus aureus (SA) is a major causative agent of nosocomial infections (1). Also, SA causes respiratory infections as well as skin problems and food poisoning. Due to increased resistance, SA is a growing public health challenge (2). Methicillin-resistant Staphylococcus aureus (MRSA) isolates represents a group of SA becoming resistant to several antibiotics such as erythromycin, levofloxacin, tetracycline, clindamycin, mupirocin, gentamicin, trimethoprim, and/or doxycycline, but it is usually sensitive to vancomycin (3). Most MRSA infections identify in those who have been in hospitals and other facilities, such as nursing homes and dialysis centers that are usually associated with invasive procedures or devices such as surgery, intravenous tubing, or artificial joints; hence it's also named as healthcare-associated MRSA(HA-MRSA). Nevertheless, MRSA also occurs in healthy people who haven't been hospitalized, known as community-associated MRSA (CA-MRSA), often begins with painful skin rashes. Such infections can transmit by skin-to-skin contact. Hence, students, wrestlers, childcare professionals, and people living in crowded places are among those who are at increased risk of CA-MRSA (4). The resistance of SA to methicillin is because of the presence of the mecA gene (5). It's estimated that the mortality rate of invasive MRSA in the United State is about 25\% (6). For example, in the city of New York, its mortality rate during the period of 2002 - 2007 has been estimated as 30\% (7). Because of increased antimicrobial resistance, Scientists are looking for new therapeutic inter- 
ventions, with a particular emphasis on bacteriophages as a viable option in treating bacterial infections either in tandem with or as a substitution for antibiotics $(7,8)$. Methicillin has been used to treat SA infections, despite increasing resistance of many species to methicillin, and to find alternative therapeutic interventions; in the present study, methicillin was selected as the target antibiotic.

Bacteriophage, also named phage, is a group of viruses that infect and replicate inside the host bacteria and finally lyse it. To penetrate the host cell, the phage requires a specific receptor (9). Nevertheless, after disruption of the host cells, phages also disappear. Phages attack a wide range of cells (10). It's a long time that the idea of phage therapy, using phages to treat infections caused by bacteria, is pursuing $(11,12)$. In some Eastern European countries, phages are using to treat bacterial infections $(13,14)$. For example, Jensen et al. (15) discovered a new lytic phage against MRSA and then purified it to eliminate human MRSA from hard fabrics and surfaces. The main reason for using phage cocktails is the extent of their activity, compared to using a single phage. Besides, they can affect different bacterial species and maintain their activity in different environmental conditions. Combining phages can also affect many bacterial strains that may be responsible for similar diseases. In general, they have a greater potential for treating infections than single phage isolates (16).

\section{Objectives}

Since multi-drug resistance has significantly increased in many SA isolates, the current study aimed to isolate phages that have a lytic effect on isolated methicillinresistant $S$. aureus from clinical human samples.

\section{Methods}

\subsection{Phage Isolation and Purification}

A modified standard method was used to enrich and isolate bacteriophages. In brief, $50 \mathrm{~mL}$ of urban sewage in Isfahan, Iran, was centrifuged at $8000 \mathrm{~g}$ for 10 minutes at $4{ }^{\circ} \mathrm{C}$. Fifteen milliliter of the supernatant was added to $50 \mathrm{~mL}$ of SA and cultured overnight in nutrient broth, followed by 24 hours of incubation at $37^{\circ} \mathrm{C}$ to the multiplication of possible staphlophages. Residual bacterial cells and debris were removed by centrifugation (8000 g, $10 \mathrm{~min}$, and $4^{\circ} \mathrm{C}$ ) and filtration with a $0.22 \mu \mathrm{m}$ Millipore filter. To confirm the presence of bacteriophage in filtrate, $100 \mu \mathrm{L}$ of overnight-cultured SA was mixed with $10 \mathrm{~mL}$ of soft brain heart infusion (BHI) broth (Darmstadi, Germany). Then, the solution was covered on the surface of $1.5 \%$ solid BHI agar (Unipath LTD, England) until solidify for 30 minutes.
Afterward, $10 \mu \mathrm{L}$ of the filtrate was spotted on the surface and incubated at $37^{\circ} \mathrm{C}$ overnight. Phage lytic effects on bacteria were confirmed by the appearance of transparent zones (plaques) on the media (17).

\subsection{Phage Characterization by Transmission Electron Mi- croscopy}

Phage morphology was determined by transmission electron microscopy (TEM) (Philips, Netherlands) of the purified phage particles. The surface of a carbon-coated copper grid was covered by staphylophages, and then negative staining with $2 \%(\mathrm{w} / \mathrm{v})$ uranyl acetate was performed. The stained grids were dried with air and visualized at an accelerating voltage of $100 \mathrm{kw}$ (17). Based on morphological characteristics, phages were identified in the family level of classification and nomenclature.

\subsection{Phage Cocktail Preparation}

For phage cocktail preparation, $200 \mu \mathrm{L}$ of each isolated phage from different families was added to $100 \mu \mathrm{L}$ of overnight-cultured SA isolates (17).

\subsection{Sample collection for isolation of S. aureus}

The samples were collected from blood, sputum, urine, ear, eyes, nose, abscess, pleural fluid, peritoneal fluid, broncho-alveolar lavage (BAL), trachea, throat, wound, secretions of patients, and the hospital environment during 2018 - 2019. SA was detected in the samples collected from two main teaching hospitals (AL-Zahra and Namazi) in Isfahan and Shiraz (Iran), respectively.

Samples were put in a nutrient broth medium (Scharlau Chemie, S.A.), and transferred to the laboratory. Then, samples were cultured on the surface of Muller Hinton agar and blood agar (Unipath LTD, England), followed by $24 \mathrm{~h}$ of incubation at $37^{\circ} \mathrm{C}$. SA isolates were identified by colony morphology, Gram staining, and results of biochemical assays, including the production of DNase, coagulase, and catalase (17). The presence of SA was confirmed by the polymerase chain reaction (PCR) method.

\subsection{Antimicrobial Susceptibility}

The antibiotic susceptibility profile of S. aureus isolates against methicillin $(5 \mu \mathrm{g})$, clindamycin $(20 \mu \mathrm{g})$, erythromycin $(15 \mu \mathrm{g})$, cefoxitin (5 $\mu \mathrm{g})$, ciprofloxacin $(5 \mu \mathrm{g})$, tetracycline $(30 \mu \mathrm{g})$, and gentamycin (10 $\mu \mathrm{g}$ ) (Padtan Teb, Iran) was evaluated by the Kirby-Bauer disk diffusion method according to clinical and laboratory standards institute (CLSI) guidelines. After $24 \mathrm{~h}$ of incubation at $37^{\circ} \mathrm{C}$, the diameters of the zones of inhibition around the disc was compared to the interpretative criteria recommended in the CLSI guidelines. The bacterial suspension of each 
sample was made and compared with the 0.5 McFarland turbidity standard. The bacterial suspension was cultured on Mueller-Hinton agar (Unipath LTD, England) plates and then incubated at $35^{\circ} \mathrm{C}$ for 18 hours. Afterward, the zones of inhibition were measured according to the CLSI proto$\operatorname{col}(18)$.

\subsection{DNA Extraction}

To find SA and mecA gene, the bacterial genomic DNA extraction was performed using the boiling method. Few colonies of pure isolated bacteria were placed into a microtube containing $100 \mu \mathrm{L}$ of double-distilled water and then were heated at $100^{\circ} \mathrm{C}$ for 10 minutes. The solution was centrifuged at $7000 \mathrm{~g}$ for $10 \mathrm{~min}$ at $4^{\circ} \mathrm{C}$ and the supernatant that containing bacterial genomic DNA was used for PCR assay (19).

\subsection{PCR Test}

The PCR assay targets the Staphylococcus genusspecific 16S rRNA gene with forwarding staph primer: 5'AACTCTGTTATTAGGGAAGAACA-3' and reverse staph primer: 5'-CCACCTTCCTCCGGTTTGTCACC-3' was performed to confirm the isolates (20).

DNA was amplified by the PCR method in a total volume of $25 \mu \mathrm{L}$, containing $1.5 \mathrm{mM} \mathrm{MgCl}$, $30 \mathrm{mM}$ dNTPs, 2 units of Taq DNA-polymerase, 1X PCR buffer (50 mM $\mathrm{KCl}, 10 \mathrm{mM}$ Tris- $\mathrm{HCl}), 0.24 \mathrm{mM}$ primer, and $50 \mathrm{ng}$ DNA. The presence of the mecA gene was detected by PCR with previously designed primers include forward primer: 5'GTAGAAATGACTGAACGTCCGATGA-3' and reverse primer: 5'-CCAATTCCACATTGTTTCGGTCTAA-3' (20).

Thermal cycling condition for PCR process was as follow $94^{\circ} \mathrm{C}$ for 3 minutes; 30 cycles at $94^{\circ} \mathrm{C}$ for 30 seconds, $55^{\circ} \mathrm{C}$ for 30 second, and $72^{\circ} \mathrm{C}$ for 45 seconds. The final extension was at $72^{\circ} \mathrm{C}$ for 5 minutes. PCR product $(5 \mu \mathrm{L})$ was then analyzed by agarose gel electrophoresis method after staining with Safe Stain and visualized by UV(21).

\subsection{Determination of Phage Cocktail Effects}

To evaluate the phage cocktail effects against SA strains, the prepared mixture was tested using the double-layer agar method (DLAM). Plates were incubated at $37^{\circ} \mathrm{C}$ for 24 hours and observed for plaque formation (17).

\subsection{Statistical Analysis}

Data were analyzed by descriptive analysis and analyzed in SPSS version 19.

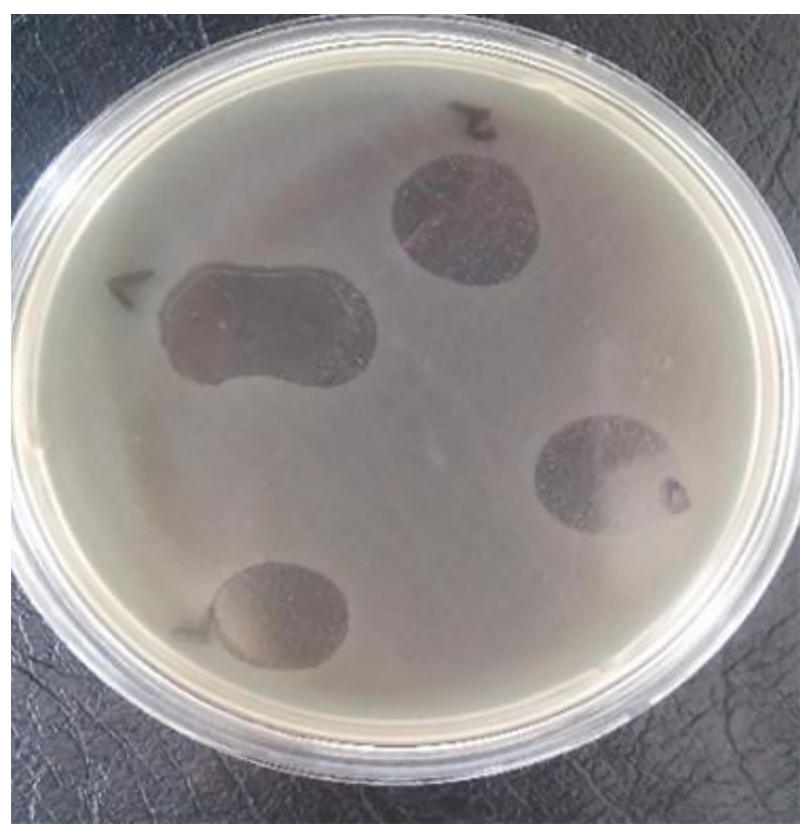

Figure 1. Clear plaques show the presence of lytic staphylophages

\section{Results}

\subsection{Phage Isolation and Identification}

Lytic staphylophages were recognized by observing clear plaques on the double-layer plates (Figure 1).

Four types of staphylophages were recognized by TEM and identified according to morphological characteristics (Springer index of viruses book) (22). The first type had a head with $30 \mathrm{~nm}$ width and $30 \mathrm{~nm}$ length, which was from the Tectiviridae family (Figure 2). The second phage had a head with $51 \mathrm{~nm}$ in width and $57 \mathrm{~nm}$ in length, a $60 \mathrm{~nm}$ tail, and a $15 \mathrm{~nm}$ endplate. This phage was from the Myoviridae family(Figure 3). For the other two phages, the width of the head was 30 and 33, and its length was 31 and $33 \mathrm{~nm}$, while the tail was 78 and $160 \mathrm{~nm}$ tail, respectively. These phages were from the Siphoviridae family (Figures 4 and 5).

\subsection{Antimicrobial Susceptibility Test and PCR}

Based on the results of antibiotic susceptibility tests, 123 (92.4\%) bacterial isolates (out of 133 Staphylococcus aureus isolates) were MRSA. Antimicrobial susceptibility profiles and the existence of the mecA gene are presented in Table 1. According to the PCR results, 118 (88.7\%) samples were positive for the mecA gene (Figure 6).

\subsection{Staphylophage Cocktails and Phage Sensitivity Test}

Two phage cocktails were prepared by mixing four types of staphylophage. Cocktail 1 contained phages VB- 


\begin{tabular}{|c|c|c|c|c|}
\hline Antibiotic & Sensitive, No. (\%) & Intermediate, No. (\%) & Resistant, No. (\%) & No. (\%) \\
\hline Methicillin & $3(2.3)$ & $7(5.3)$ & $123(92.4)$ & \\
\hline Clindamycin & $74(55.6)$ & $15(11.2)$ & $44(33.0)$ & \\
\hline Erythromycin & $74(55.6)$ & $17(12.7)$ & $42(31.5)$ & \\
\hline Cefoxitin & $50(37.6)$ & $21(15.7)$ & $62(46.6)$ & \\
\hline Ciprofloxacin & $50(37.6)$ & $15(11.2)$ & $68(51.1)$ & \\
\hline Tetracycline & $58(43.6)$ & $17(12.7)$ & $58(43.6)$ & \\
\hline Gentamycin & $91(68.4)$ & $8(6.0)$ & $34(25.5)$ & \\
\hline mecA gene & & & & $118(88.7)$ \\
\hline
\end{tabular}

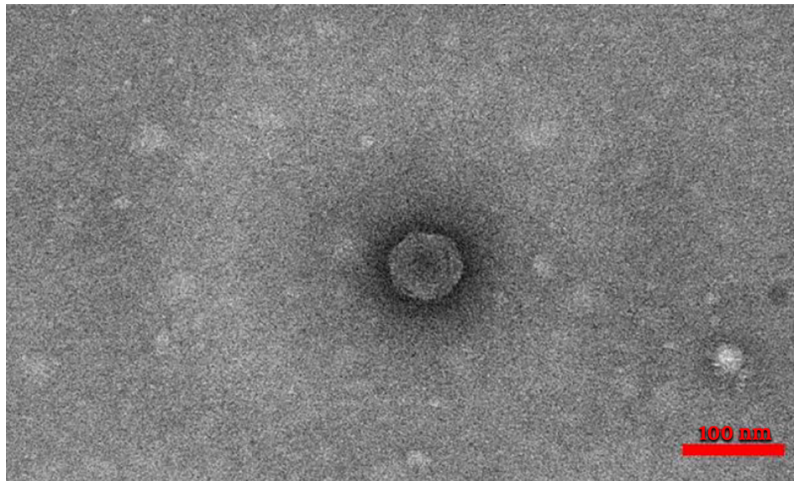

Figure 2. Electron micrograph of the phage VB-StaphT-Isf 43 belonged to the Tectiviridae family with a head size of $30 \times 30 \mathrm{~nm}$.

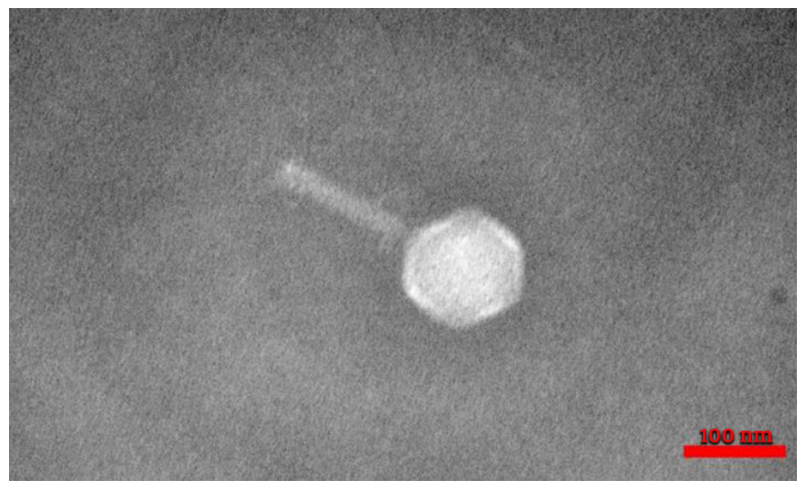

Figure 3. Electron micrograph of the phage VB-StaphM-Isfio2 belonged to the $M y$ oviridae family with a head size of $57 \times 50 \mathrm{~nm}$, a tail of $60 \mathrm{~nm}$, and an endplate of 15 $\mathrm{nm}$, with attached fibers.

StaphT-Isf43 from the Tectiviridae family and VB-StaphSIsf65 phage from the Siphoviridae family. Cocktail 2 contained VB-StaphM-Isfio2 phage from the Myoviridae family and VB-StaphS-Isf 29 from the Siphoviridae family. The results of the phage susceptibility test showed that 44 SA iso-

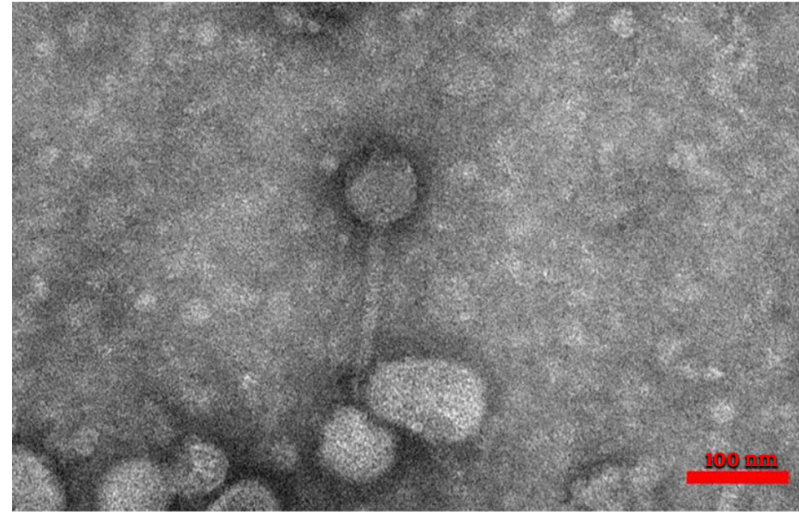

Figure 4. Electron micrograph of the phage VB-StaphS-Isf65 belonged to the Siphoviridae family with a head size of $31 \times 31 \mathrm{~nm}$, a tail of $78 \mathrm{~nm}$.

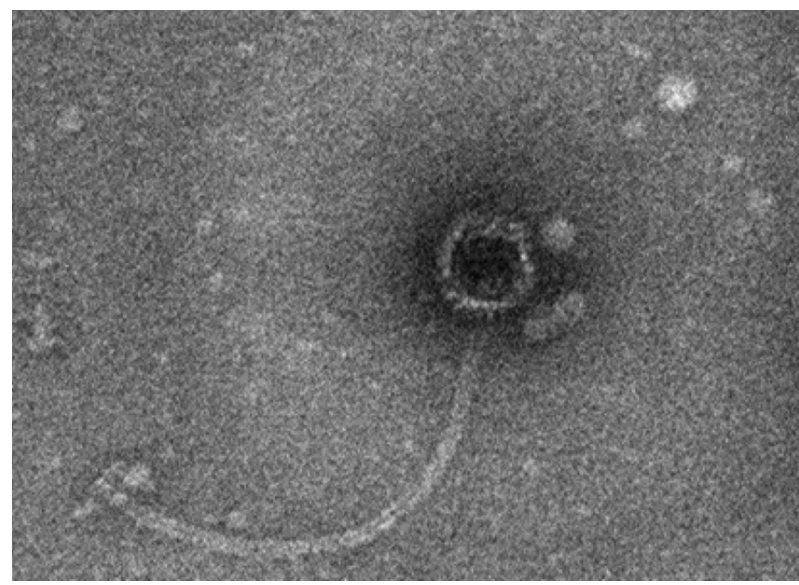

Figure 5. Electron micrograph of the phage VB-StaphS-Isf 29 belonged to the Siphoviridae family with a head size of $30 \times 33 \mathrm{~nm}$, a tail of $160 \mathrm{~nm}$.

lates were lysed by two cocktails, and plaques were produced. Cocktails 1 and 2 lysed 19 (14.2\%) and 25 (18.7\%) MRSA 


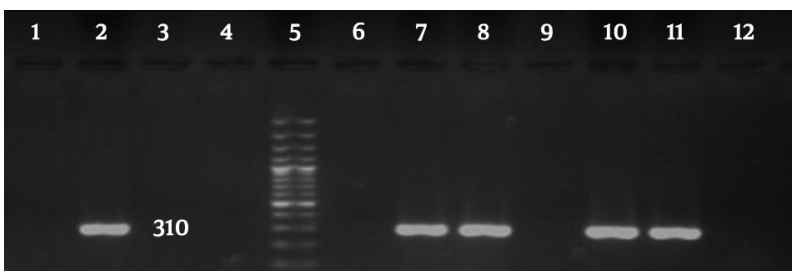

Figure 6. Detection of the mecA gene in SA isolates by running of PCR products on $1.5 \%$ agarose gel electrophoresis. Lane 1 is negative control and lane 2 is positive control ( $310 \mathrm{bp}$ ), lanes 7, 8, 10, and 11 are positive samples, and lane 5 is $100 \mathrm{bp}$ DNA marker.

isolates, respectively.

\section{Discussion}

In the present study, the resistance of clinical isolates of SA to methicillin was the highest reported rate in Iran, which indicates the growing trend during recent years. Faridi et al. (23) reported a low prevalence (10\%) for MRSA among SA isolates. While Shokri et al. (2) reported a prevalence of about $57.8 \%$ in 45 SA isolates for methicillin resistance, and Rahimi et al. (24), 68\% in 50 SA isolates. Safdari et al. (25) reported that $30 \%$ of 68 SA isolates were positive for beta-lactamase. Jafari-Sales et al. (26) reported that 75 (out of the 100 SA isolates) were methicillin-resistant, which $68 \%$ of them harbored the mecA gene. In the present study, the observed antibiotic resistance is the highest amount that is reported until now in Iran. Moreover, the prevalence of the mecA gene is the highest reported value in Iran. This difference can be attributed to factors such as geographical locations and the source of SA isolates. While phage therapy is a newly adopted approach in Iran, its application as a potential intervention to treat infections has a long history in some Eastern European countries. Phage susceptibility tests are used to identify the type of phages in some genus of bacteria, such as Brucella species. As mentioned above, most of the clinical isolates of S. aureus have become resistant to several drugs, including methicillin. Therefore, this study evaluated the antibacterial effect of staphylophages in eliminating the MRSA isolates as an alternative way to antibiotic therapy. Based on the findings, four types of staphylophage from Siphoviridae, Myoviridae, and Tecticoviridae families had a relatively good effect on MRSA clinical isolates. In this study, 44 samples (out of 133) were lysed by two phage cocktails, so that cocktails 1 and 2 lysed 19 (14.2\%) and 25 samples (18.7\%), respectively. Phage cocktails could lyse MRSA strains. Phage cocktail 2 (Myoviridae and Siphoviridae) had the highest lytic effect, which suggests its effectiveness. However, it needs further investigation in tissue cultures and animal models. Although some researchers reported that staphylophages could lyse MRSA isolates, but these effects may not be observed in isolates obtained from other geographical areas. In Eastern European countries and the former Soviet Union the potential antimicrobial effects of phage therapy are wellproved (27). Jensen et al. (15) isolated a new lytic phage against MRSA to eliminate infections caused by bacteria on the surfaces.

They realized that the fLizAnk phage had no toxic effect on fibroblast cell culture, and the antibacterial effect of phage against MRSA was presented in cell culture. Since the fLizAnk phage showed antibacterial activity against MRSA strains and had no cytotoxic effect against mammalian cells, it may be safe lonely or with a phage cocktail for the treatment of skin infection caused by SA. In another study by Jensen et al. (15) 12 phages were isolated and could replicate in human samples and/or MRSA isolates and eliminated the infection. They also investigated the advantages of some phages to decontaminate fomites (glass and cloth) and found a significant reduction in colonyforming units of MRSA following treatment with phage, including tests of a phage cocktail against MRSA isolates. In another study, Trigo et al. (28) used bacteriophages as an alternative antimicrobial treatment to control bacterial infections. Lehman et al. (29) have described the design and preclinical development of AB-SA01 (lytic myoviruses), a fixed-composition. Finally, they found that the inherent characteristics of AB-SA01 component phages met the regulatory and generally accepted criteria for human use, and reported that the presented preclinical data support the production under good manufacturing practices and phase 1 clinical studies with AB-SA01.

In another study, Abo-Elmaaty et al. (30) isolated a phage from the Myoviridae family and reported its antibacterial and anti-biofilm properties against SA. Jo et al. (31) designed a way to show the synergistic antimicrobial effect of phages combined with antibiotics against SA. Based on the results, the combined treatment of phages and antibiotics can be used to meliorate antimicrobial impression against antibiotic-resistant bacteria. Chan et al. (32) reported that phage therapy can be used to treat bacterial infections in humans, domestic animals, and even biocontrol in foods. Fabijan et al. (33), in a single-arm non-comparative trial on 13 patients with severe SA infections who were intravenously administered three Myoviridae bacteriophages (AB-SA01) as adjunctive therapy, reported no adverse reactions. This indicates that intravenous administration of AB-SA01 is safe in patients with severe SA infections, including infective endocarditis and septic shock. 


\subsection{Conclusions}

This study demonstrated that some staphylophages isolated from urban sewage have appropriate effectiveness against clinical MRSA pathogens. Nevertheless, this issue should be further investigated in future studies on the inhibitory effects of staphylophages on other bacteria and even in vivo experiments.

\section{Footnotes}

Authors' Contribution: All authors contributed equally to planning and performing this study.

Clinical Trial Registration Code: We have no clinical trial Conflict of Interests: The authors declare no conflict of interest.

Ethical Approval: This research was approved by the Ethics Committee of the Research Council of the Shahrekord University, Faculty of Veterinary Medicine with ethics code: 170.1336 .

Funding/Support: The study was funded by the Higher Education of Shahrekord University.

Informed Consent: No patient was involved in this study, and samples were collected from wastewater and laboratory collection.

\section{References}

1. AhmadI Z, Tajbakhsh E, Momtaz HASSAN. Detection of the antibiotic resistance pattern in Staphylococcus aureus isolated from clinical samples obtained from patients hospitalised in Imam Reza hospital, Kermanshah. J Microb Wld. 2014;6(17):209-311.

2. Shokri R, Salouti M, Sorouri ZR, Heidari Z. Frequency of meticillin resistant Staphylococcus aureus strains isolated from clinical samples in Mousavi Hospital, Zanjan, and recognition mec A gene using PCR. J Microb Wld. 2014;7(18):58-65.

3. McDougal LK, Fosheim GE, Nicholson A, Bulens SN, Limbago BM, Shearer JE, et al. Emergence of resistance among USA300 methicillinresistant Staphylococcus aureus isolates causing invasive disease in the United States. Antimicrob Agents Chemother. 2010;54(9):380411. doi: 10.1128/AAC.00351-10. [PubMed: 20585117]. [PubMed Central: PMC2934979].

4. Gorwitz RJ, Jernigan DB, Jernigan JA. Strategies for clinical management of MRSA in the community; summary of an experts' meeting convened by the Centers for Disease Control and Prevention. CDC. 2006.

5. Deurenberg RH, Stobberingh EE. The evolution of Staphylococcus aureus. Infect Genet Evol. 2008;8(6):747-63. doi: 10.1016/j.meegid.2008.07.007. [PubMed: 18718557].

6. Dantes R, Mu Y, Belflower R, Aragon D, Dumyati G, Harrison LH, et al National burden of invasive methicillin-resistant Staphylococcus aureus infections, United States, 2011. JAMA Intern Med. 2013;173(21):19708. doi:10.1001/jamainternmed.2013.10423. [PubMed: 24043270].

7. Mann NH. The potential of phages to prevent MRSA infections. Res Microbiol. 2008;159(5):400-5. doi: 10.1016/j.resmic.2008.04.003. [PubMed: 18541414].
8. Viertel TM, Ritter K, Horz HP. Viruses versus bacteria-novel approaches to phage therapy as a tool against multidrug-resistant pathogens. J Antimicrob Chemother. 2014;69(9):2326-36. doi 10.1093/jac/dku173. [PubMed: 24872344].

9. Naser A, Azizi Jalilian F, Azizian R, Askari H, Pakzad I. The Use of MRSA phage as a super specialized antibiotic against lethal methicillinresistant Staphylococcus aureus. J Ilam Uni Med Sci. 2014;22(4):171-9.

10. Skurnik M, Strauch E. Phage therapy: facts and fiction. Int J Med Microbiol. 2006;296(1):5-14. doi: 10.1016/j.ijmm.2005.09.002. [PubMed 16423684].

11. Sulakvelidze A, Alavidze Z, Morris JJ. Bacteriophage therapy. Antimicrob Agents Chemother. 2001;45(3):649-59. doi: 10.1128/AAC.45.3.649659.2001. [PubMed: 11181338]. [PubMed Central: PMC90351].

12. Burrowes B, Harper DR, Anderson J, McConville M, Enright MC. Bacteriophage therapy: potential uses in the control of antibioticresistant pathogens. Expert Rev Anti Infect Ther. 2011;9(9):775-85. doi: 10.1586/eri.11.90. [PubMed: 21905786].

13. Kutter E, Sulakvelidze A. 14 Bacteriophage Therapy in Humans. Bacteriophages: biology and applications. London, New York: Crc press; 2004.

14. Cisek AA, Dabrowska I, Gregorczyk KP, Wyzewski Z. Phage therapy in bacterial infections treatment: one hundred years after the discovery of bacteriophages. Curr Microbiol. 2017;74(2):277-83. doi: 10.1007/s00284-016-1166-x. [PubMed: 27896482]. [PubMed Central: PMC5243869].

15. Jensen KC, Hair BB, Wienclaw TM, Murdock MH, Hatch JB, Trent AT, et al. Isolation and host range of bacteriophage with lytic activity against methicillin-resistant staphylococcus aureus and potential use as a fomite decontaminant. PLoS One. 2015;10(7). e0131714. doi: 10.1371/journal.pone.0131714. [PubMed: 26131892]. [PubMed Central: PMC4488860]

16. Chan BK, Abedon ST. Phage therapy pharmacology phage cocktails. Adv ApplMicrobiol.2012;78:1-23. doi:10.1016/B978-0-12-394805-2.000014. [PubMed: 22305091].

17. Ghasemi SM, Bouzari M, Emtiazi G. Preliminary characterization of lactococcus garvieae bacteriophage isolated from wastewater as a potential agent for biological control of lactococcosis in aquaculture. Aquac Int. 2014;22(4):1469-80. doi:10.1007/s10499-014-9760-Z.

18. Cockerill FR, Wikler MA, Alder J, Dudley MN, Eliopoulos GM, Ferraro MJ, et al. Performance standards for antimicrobial susceptibility testing: twenty-second informational supplement. Clin Lab Stand Inst. 2012;32(3):M100-S22.

19. Kazemnia A, Ahmadi M, Dilmaghani M. Antibiotic resistance pattern of different Escherichia coli phylogenetic groups isolated from human urinary tract infection and avian colibacillosis. Iran Biomed J. 2014;18(4):219-24. doi: 10.6091/ibj.1394.2014. [PubMed: 25326020] [PubMed Central: PMC4225061].

20. Zhang K, Sparling J, Chow BL, Elsayed S, Hussain Z, Church DL, et al. New quadriplex PCR assay for detection of methicillin and mupirocin resistance and simultaneous discrimination of Staphylococcus aureus from coagulase-negative staphylococci.JClin Microbiol. 2004;42(11):4947-55. doi: 10.1128/JCM.42.11.4947-4955.2004. [PubMed 15528678]. [PubMed Central: PMC525205].

21. McClure JA, Conly JM, Lau V, Elsayed S, Louie T, Hutchins W, et al Novel multiplex PCR assay for detection of the staphylococcal virulence marker Panton-Valentine leukocidin genes and simultaneous discrimination of methicillin-susceptible from -resistant staphylococci. J Clin Microbiol. 2006;44(3):1141-4. doi: 10.1128/JCM.44.3.11411144.2006. [PubMed: 16517915]. [PubMed Central: PMC1393128].

22. Tidona C, Darai G. The Springer index of viruses. New York: Springer Science \& Business Media; 2011. doi:10.1007/978-0-387-95919-1.

23. Faridi A, Kareshk AT, Fatahi-Bafghi M, Ziasistani M, Ghahraman MRK Seyyed-Yousefi SZ, et al. Detection of methicillin-resistant Staphylococcus aureus (MRSA) in clinical samples of patients with external ocular infection. Iran J Microbiol. 2018;10(4):215. 
24. Rahimi HM, Mirzaie AMIR, Salehzadeh A. Frequency of methicillin resistant (mecA) and panton-valentine leucocidin (pvl) genes among Staphylococcus aureus isolates recovered from clinical samples of Rasht hospitals. J Microb Wld. 2016;9(26):34-43.

25. Safdari H, Safdari A, Navaei N, Hashemzadeh M. Evaluation Resistance prevalence of Staphylococcus Aureus $\beta$-Lactamase from Nasal of Emergency Staff in Ghaem Hospital of Mashhad 1397. med j mashhad univ med sci. 2020;63:2334-9. doi: 10.22038/MIMS.2020.16016.

26. Jafari-Sales A, Jafari B. Evaluation of the Prevalence of mec A Gene in Staphylococcus aureus Strains Isolated from Clinical Specimens of Hospitals and Treatment Centers. Pajouhan Scientific J. 2019;17(3):41-7. doi: 10.29252/psj.17.3.41.

27. Biswas B, Adhya S, Washart P, Paul B, Trostel AN, Powell B, et al. Bacteriophage therapy rescues mice bacteremic from a clinical isolate of vancomycin-resistant Enterococcus faecium. Infect Immun. 2002;70(1):204-10. doi: 10.1128/iai.70.1.204-210.2002. [PubMed 11748184]. [PubMed Central: PMC127648].

28. Trigo G, Martins TG, Fraga AG, Longatto-Filho A, Castro AG, Azeredo J, et al. Phage therapy is effective against infection by Mycobacterium ulcerans in a murine footpad model. PLoS Negl Trop Dis. 2013;7(4). e2183. doi: 10.1371/journal.pntd.0002183. [PubMed
23638204]. [PubMed Central: PMC3636042].

29. Lehman SM, Mearns G, Rankin D, Cole RA, Smrekar F, Branston SD, et al. Design and preclinical development of a phage product for the treatment of antibiotic-resistant staphylococcus aureus infections. Viruses. 2019;11(1). doi: 10.3390/v11010088. [PubMed: 30669652]. [PubMed Central: PMC6356596].

30. Abo-Elmaaty S, El Dougdoug NK, Hazaa MM. Improved antibacterial efficacy of bacteriophage-cosmetic formulation for treatment of Staphylococcus aureus in vitro. Ann Agric Sci. 2016;61(2):201-6. doi: 10.1016/j.aoas.2016.08.002.

31. Jo A, Ding T, Ahn J. Synergistic antimicrobial activity of bacteriophages and antibiotics against Staphylococcus aureus. Food Sci Biotechnol. 2016;25(3):935-40. doi: 10.1007/s10068-016-0153-0. [PubMed: 30263357]. [PubMed Central: PMC6049171].

32. Chan BK, Abedon ST, Loc-Carrillo C. Phage cocktails and the future of phage therapy. Future Microbiol. 2013;8(6):769-83. doi: 10.2217/fmb.13.47. [PubMed: 23701332].

33. Fabijan AP, Lin RC, Ho J, Maddocks S, Zakour NLB, Iredell JR. Safety of bacteriophage therapy in severe Staphylococcus aureus infection. $\mathrm{Na}$ ture microbiol. 2020;5(3):465-72. 